\title{
Field dependence-independence in the development of referential communication
}

\author{
RAFE AL-NESIR \\ Mu'tah University, Mu'tah, Jordan \\ and \\ VERNE KEENAN and PHILIP LANGER \\ University of Colorado, Boulder, Colorado
}

\begin{abstract}
Children do not easily learn to evaluate verbal messages for ambiguity, and recent investigation has begun to find variables that improve our understanding of the development of this referential communication ability. On the basis of their scores on the Children's Embedded Figures Test (CEFT), 72 first- and third-graders were assigned, within grades, to three levels of field dependenceindependence. In each of the resulting groups, the subjects were assigned randomly to a picturecontext or a speak-only group for a test of message evaluation. Message evaluation was better for the older children, for the more field-independent children, and for the picture-context condition. None of the interactions was reliable. Results are discussed with respect to their relevance for the development of critical thinking in language reception and development of literacy.
\end{abstract}

Literacy is a major concern today in all parts of the world, and an important factor in the development of children's literacy is the ability to judge the adequacy of verbal communication. Much of what we hear and read is referentially ambiguous, and we have learned to ask speakers to repeat or clarify their messages. Children do not easily learn to request such clarification or even to understand that messages may be ambiguous. If we show a child a red knife and a red spoon and say, "Show me the red one," it is important for the child to notice the inadequacy of the request as given. Studies of referential communication, or message evaluation, have shown that preschool children do not recognize such inadequacies, and that primary-grade children appear to notice that an instruction or question is unclear, but do not know what is wrong with it or what to do about it (Beal, 1987). We can expect that language education at home and in the early school years will be enhanced as we better understand the abilities and experiences that influence children's message-evaluation performance.

Many factors in the child and in the context, including the age of the listener and cues from the environment, contribute to referential communication. If children believe they know the intent of the speaker, they may act on that belief rather than monitor the message for precise meaning (Beal, 1987). Furthermore, performance is better if the message is in written or pictorial form, and therefore available for inspection. It is generally agreed that

This article is based on a paper given at the meeting of the Western Psychological Association, held in Reno, Nevada, in 1989. Correspondence should be addressed to Philip Langer, Department of Psychology, Muenzinger Psychology Building, Campus Box 345, Boulder, CO 80309-0345. older children are better than younger ones at critically monitoring the messages they hear, and that brighter children do better than the less bright. Some researchers have attempted to relate cognitive styles to message evaluation. There is recent evidence that more reflective children show better communication monitoring than do more impulsive children (Pratt \& Wickens, 1983). These findings suggest that other characteristics of listeners may be related to this important metalinguistic ability. The other major cognitive style in the literature is field dependenceindependence; using a different paradigm with adults, comprehension of ambiguous sentences has been found to be linked to field dependence-independence (Lefever \& Ehri, 1976). The aim of the present investigation was to determine whether field-independent children, like adults, are more capable of message evaluation than their field-dependent peers. The study also replicated age and visual-context effects known in the literature, and extended these phenomena to children in Jordan.

\section{METHOD}

The materials used included the Children's Embedded Figures Test
(CEFT), a modification of the Embedded Figures Test (Lefever \& Ehri,
1976), to assess field dependence, the Cognitive Abilities Test (CogAT),
and the visual-context pictures with practice materials as needed. The
dependent variable was assessed by the Message Evaluation Test (MET),
a set of 16 items adapted from materials used by Pratt and Wickens
(1983). For 8 items, the question was ambiguous; for the other 8 , the
question was clear. The sentence "Jane has a red pencil and Jim has
a blue pencil" may be followed by the ambiguous question, "Who has
the pencil, Jane or Jim?" or by the unambiguous question, "Who has
the blue pencil?" After each item, the child was asked if the question
was "good" or "bad." If the question was ambiguous, the child was
to call it "bad" and say what was wrong with it.
The subjects were 72 children from a middle-class neighborhood in
the Irbid district of Jordan. Eighteen boys and 18 girls were first-graders, 
and 18 boys and 18 girls were third-graders, who were randomly assigned to the visual-context or the speaker-only conditions. For half of the children, the MET was presented verbally along with a pictorial representation. The pictures, which matched the verbal message, were line drawings of, for example, a boy with a car and a girl with an airplane. The other instruments used were the CogAT and the CEFT. The CogAT was included as a covariate since the differences in the cognitive abilities of primary children might be relatively large. The CEFT is a measure of the trait called field dependence-independence, or psychological differentiation, the ability to analyze a situation or to separate a part from the whole. The test involves a house, for example, presented as a cutout form, and a larger picture or design in which the house is embedded. There were 25 such designs in the form used, and all of the materials were translated into Arabic.

The CogAT was administered to small groups, and the CEFT was administered individually. The CEFT scores were divided into thirds within grade-by-gender groups. Children in each of the resulting 12 groups were randomly assigned to the picture context or the control condition for the individually administered MET. Responses were taperecorded and later scored for the numbers of correct evaluations.

\section{RESULTS AND DISCUSSION}

The principal issue of this investigation was the relationship of message evaluation to field dependenceindependence. The MET scores were reliably different for the three CEFT levels $[F(2,60)=11.8, p<.01]$. Subjects with higher CEFT scores are field-independent, and the MET differences were predicted. The effect sizes are .59 for the intermediate level and 1.40 for the high level on the CEFT. MET scores were higher for thirdthan for first-grade subjects $[F(1,60)=45.8, p<.01]$. The effect size was 1.60 . These findings agree with the developmental effects found for American samples, and was predicted. Physical context yielded a significant effect $[F(1,60)=23.5, p<.01]$; that is, the picture cues were more effective, yielding an effect size of 1.15 . There were no significant interactions. Although the verbal and quantitative scores on the CogAT were statistically significant sources of variance in the total sample, the pattern of effects provided by an analysis of covariance did not differ from that of an analysis of variance. Only the analysis of variance data are therefore reported.

These results, taken together, indicate that the general problem of message evaluation is not limited to American children and probably applies to children everywhere. Age, ability, and context facilitation effects were all replicated. In addition, it is suggested that field-independent children are generally more capable than field-dependent children in the detection of ambiguity in referential communication. The CEFT effect must, of course, be replicated using other samples of children. But, to the extent that these various effects are widespread among children of all places and cultures, it is important to consider how they may influence practice in the teaching of language. Improvement in message evaluation may be the beginning of critical thinking in all linguistic activities, and practice in message evaluation, with and without physical context cues, may be offered as a part of instruction.

\section{REFERENCES}

BEAL, C. R. (1987). Repairing the message: Children's monitoring and revision skills. Child Development, 58, 401-408.

Lefever, M. M., \& EHRI, L. C. (1976). The relationship between field independence and sentence disambiguation ability. Journal of Psycholinguistic Research, 5, 99-106.

Pratt, M. W., \& Wickens, G. (1983). Checking it out: Cognitive style and problem type in children's monitoring of text comprehension. Journal of Educational Psychology, 75, 716-726.

(Manuscript received May 23, 1990.) 